\title{
The Development and Evaluation of an Interactive System for Age Related Musculoskeletal Rehabilitation in the Home
}

\author{
Mobolaji Ayoade, Stephen Uzor, and Lynne Baillie \\ Interactive and Trustworthy Technologies Group, School of Engineering and Built \\ Environment, Glasgow Caledonian University, \\ 70 Cowcaddens Road, UK \\ \{Mobolaji.Ayoade, Stephen.Uzor, L.Baillie\}@gcu.ac.uk
}

\begin{abstract}
This paper describes a series of user studies carried out to investigate the usability, significance, and acceptance of two visualization tools designed to improve the quality of, and adherence to home-based exercise programmes for musculoskeletal rehabilitation. The core functionality of these visualization tools enabled the users to observe the optimal way to perform their exercises via a mannequin, and receive feedback on their own movements through the use of body worn sensors. Before full deployment in the home, two user studies were carried out in the laboratory, and then two in the home with seniors who had recently undergone musculoskeletal rehabilitation using a standard care paper based booklet in the home. Our key findings suggest that by using the visualization tools the participants were able to overcome the major limitations of standard care; and that these tools were considered by the users to be useful in encouraging participation in home exercise.
\end{abstract}

Keywords: Home rehabilitation, inertial motion sensors, older adults, visualizations and musculoskeletal conditions.

\section{Introduction}

Musculoskeletal conditions are the most common cause of physical disability and severe long-term pain in older adults [1]. In particular, the degeneration of muscles and joints due to age can increase a person's risk of falling; and possibly lead to osteoarthritis (excessive joint pain) in the knees or hips - with a replacement of these joints being the recommended solution. The two conditions we focus on in this paper are rehabilitation after knee surgery or after a fall. These conditions normally affect older adults or seniors. For example, in Scotland, the average age for adults undergoing knee replacement surgery is 68 years [2] and over $30 \%$ of adults over the age of 65 suffer a fall at least once a year. These impairments in muscle strength and balance can have serious repercussions with regards to the independence and confidence of older adults [3]. 
Exercise programmes are often used - both as preventive, and rehabilitative means - to help elderly patients return to functioning physical levels [4]. In hospitals and community-based rehabilitation centers, strategies involving the use of exercise for rehabilitation have been very successful [5-6]. However, in the home setting, recovery of balance and strength can be challenging, as individuals often do not adhere to rehabilitation programmes thereby making this type of intervention less effective [7-8]. In addition, the individuals sometimes do not correctly interpret the instructions and health care professionals are not aware of this lack of understanding until the patient returns to the hospital or rehabilitation clinic for a follow-up appointment.

The consequence of this lack of adherence and poor quality performance has serious implications for the health and independence of the individual. In some cases, seniors who have had a recent injurious fall may feel isolated and fearful of future falls, thereby limiting their movement and thereby actually increasing their fall risk [9-10]. In another example, people who have undergone knee replacement surgery may not recover full range of movement in the new joint(s) thereby limiting their knee function and having a negative impact on their quality of lives - if they do not exercise the joint(s) regularly as recommended by health experts [11]. In order to address these issues, it is important to identify what the short comings of standard home rehabilitation are, and investigate ways in which greater participation in home exercise can be encouraged.

One of the ways through which rehabilitation can be enhanced, we believe, is through the use of visual feedback or visualization. By visual feedback, we mean the capture of the user's movement, then presenting it in a form that is easily understandable and that indicates how well the user's movement matches the ideal movement. In previous research studies, visual feedback has been used to provide biomechanical feedback on user's performance during gait retraining [12], and the use of visual feedback to retrain balance in the elderly after a stroke [13]. However to facilitate this kind of feedback, specialized equipment and staff are often required to capture the patients' movements. Numerous studies investigating visual rehabilitation have therefore been limited to laboratory or clinical settings due to these factors [14]. To investigate the potential of visual feedback to aid rehabilitation in the home setting, there is a need to design a system capable of providing the user with meaningful feedback, while being easy to use and manage by seniors.

This paper describes a series of usability studies carried out to evaluate two distinct visualization tools to improve musculoskeletal rehabilitation for seniors using relatively cheap wireless motion capture devices. Both of these tools were designed to provide de-personalized visualizations of user movements through the use of a digital mannequin. Through the use of these systems, it is possible for the users to see the ideal range and pace of motion necessary for effective recovery, and see a visualization of their own movements during home exercise. We investigate through this research, the usability and acceptance of the visualization tools in two musculoskeletal conditions with different requirements - falls and knee replacement rehabilitation. 


\section{Related Work}

Uzor et al. [15] identified one of the barriers to the effective use of current home rehabilitation tools was the inability to maintain motivation throughout the rehabilitation programme, which usually extends over long periods, due to the passive nature of current tools such as exercise booklets and DVDs. In addition, the absence of a physiotherapist and recording of exercises performed has been reported as other factors affecting adherence to home exercise programmes [16]. As a result, there has been a growing interest in the research community to seek technological solutions to these limitations [14], [17], [18]. According to Zhou [14], however, technologies for rehabilitation have not been accepted and adopted in the home in the past, especially by seniors for the following reasons: the systems have required professional help to set them up, occupy large or fixed spaces, are quite expensive and have poor human computer interaction.

Recently, there has been a growing interest among researchers and health professionals in using commercial systems such as the Nintendo Wii as rehabilitation tools for various conditions including musculoskeletal conditions [19]. Video games also have the potential of improving motivation and adherence to rehabilitation programs by facilitating the enjoyability of physical activities but they do not necessarily promote correct performance of rehabilitation exercises as the game controllers capture users' gestures or gross movement patterns [15], [19], [20]. Consequently, researchers and game enthusiasts are currently making attempts to develop games specifically designed for rehabilitation e.g. SilverPromenade [21] and Animal feeder [22]. And although there is evidence of increasing acceptance of commercial video game technologies by seniors for rehabilitation [23], [24], as yet the usability of such systems in an autonomous home environment is yet to be reported - all the studies have focused on rehabilitation centers or outpatient hospital departments and require help to setup. Ziefle \& Röcker [25] believed that by understanding the capabilities, limitations, needs and preferences of seniors; researchers and designers could produce acceptable technologies for home rehabilitation.

Theng et al. [23] identified ease of use as one of the factors to be considered when designing technology for seniors. Although, depth cameras such as the Microsoft Kinect have the potential to be easy to use by older adults [22], camera based systems face the problem of occlusion and need they require at least a small amount of free and clear space. For example, the use of the SilverFit rehabilitation system in centres has been reported [26]. However, the system required the presence of a physiotherapist and large dedicated free space $5 \times 5 \mathrm{~m}$; and as a result would not be suitable for use in most homes. Also, given a patients injuries from their fall or knee surgery, it would not be sensible for them to start moving equipment or furniture to create free space. Another issue with regard to knee and falls patients is the need to use supports which may be between the user and the cameras while performing the exercises. For example, a knee patient must sit or lie on a bed or chair in a comfortable position while performing the exercises [27-28], therefore occlusion would occur and the system would not show the users movement correctly. 
More recently, researchers have started to explore the fusion of cameras systems and inertial motion sensors e.g. monitoring of the upper arm during eating activity after stroke [29] in order to overcome the limitations of camera based systems in handling occlusion [30]. Doyle et al. demonstrated the acceptance and usability of a home-based visualization system that uses 2 step counters and a web camera to track 3 reflective markers for a home physiotherapy program for falls prevention for seniors [18]. However, these setups would be unsuitable for providing precise movement information, which is important in maximizing physiotherapy benefits of the knee rehabilitation exercise program, although they were able to monitor the correct completion of exercises and track the number of repetitions. As with other camera based systems, the markers can easily be occluded and the cameras require sufficient free space around the user thus limiting the type of physiotherapy exercises that can be monitored. In this paper we present our visualization system that is capable of capturing finer motor movements without space constraints for two rehabilitation scenarios i.e. falls prevention and knee replacement rehabilitation.

\section{Technology}

We organized a series of user design workshops actively involving older adults in the initial design of the visualization tools [15], [31]. We also consulted falls experts and physiotherapists in order to obtain their opinions on the factors to consider in the delivery of useful feedback and advice to fallers and knee replacement patients undergoing home rehabilitation. It was important for us to identify the way in which the visualizations could improve the rehabilitation process, as well as the challenges that elderly users may have when using them and the novel technology. In this section, we describe the visualization tools and technologies that were evaluated.

\subsection{Inertial Sensors}

We developed inertial sensors that could be easily worn by the users. An orientation sensor fusion algorithm was implemented using the embedded $\mathrm{C}$ language, to translate the readings of the individual inertial sensor components (accelerometer, a rate

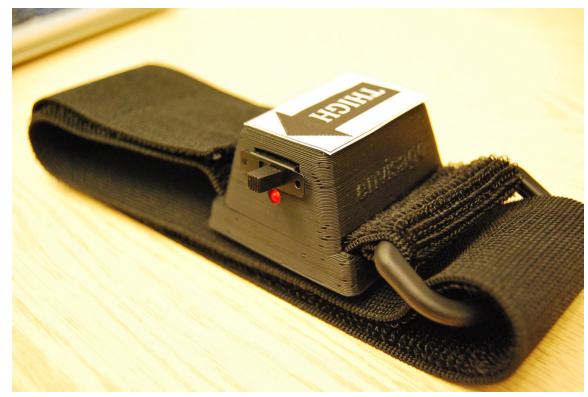

Fig. 1. The inertial motion sensor 
gyroscope and a magnetometer) and output the orientation of the sensor in world space. While the accelerometer and gyroscope were needed to calculate the 3dimensional orientation of the sensor, the magnetometer was included to reduce the effects of 'sensor drift' (i.e. the gradual wandering of gyroscope values over time) on the accuracy of the readings. The sensor data is sent wirelessly to a data receiver attached to a computer that uses the data to feedback user movements through the visualization software. Other significant features of the sensors included: a large switch to make it easy for elderly users to switch the sensor on, and a bright LED light to let them know when it was switched on.

\subsection{Visualization Tools (VT)}

For this research, two variations of the visualization tool were developed for each of the musculoskeletal conditions being investigated because the main requirements for both were different. At a base level, however, the visualization tools for both falls and knee replacement were somewhat similar e.g. they were both designed to provide visual feedback to users during home exercise based on the individual requirements of the various conditions. These requirements are highlighted in Table 1.

Table 1. Visualization Tool (VT) Requirements

\begin{tabular}{|c|c|}
\hline Requirement & Solution provided by the VT \\
\hline $\begin{array}{l}\text { 1. Show the user how and where on the } \\
\text { body the sensors should be placed }\end{array}$ & $\begin{array}{l}\text { Diagrams and text instructions showing the users } \\
\text { the correct procedure }\end{array}$ \\
\hline $\begin{array}{l}\text { 2. Show the user how the exercise } \\
\text { should be done (emphasize quality of } \\
\text { motion) }\end{array}$ & $\begin{array}{l}\text { a. An animated 'guide mannequin' highlighting the } \\
\text { correct pace and range of motion for each exercise } \\
\text { b. Text-based instructions describing important } \\
\text { advice about each exercise }\end{array}$ \\
\hline $\begin{array}{l}\text { 3. Show the user how many repetitions } \\
\text { have been achieved during exercise }\end{array}$ & $\begin{array}{l}\text { A text-based repetition counter showing the number } \\
\text { of repetitions done, and how many more to do }\end{array}$ \\
\hline $\begin{array}{l}\text { 4. Feedback to the user on their own } \\
\text { performance during exercise }\end{array}$ & $\begin{array}{l}\text { a. A user mannequin showing the users' movements } \\
\text { as captured by the inertial sensors } \\
\text { b. A colored graphic showing the quality of each } \\
\text { range of movement while the user moves the af- } \\
\text { fected leg (knee VT only) } \\
\text { c. A colored icon for each repetition showing how } \\
\text { well each repetition was done (knee VT only) }\end{array}$ \\
\hline 5. Show a chart of weekly progress & $\begin{array}{l}\text { Charts showing the average number of completed } \\
\text { exercises and the quality of the range of motion } \\
\text { associated with the exercises (knee VT only) }\end{array}$ \\
\hline
\end{tabular}

The software was written in C++, QT (to allow for flexible scripting) and Ogre (a $3 \mathrm{D}$ animation engine) and at the base level displays movement information using a stick figure, as well as other text based information [31-32]. 


\subsection{Falls Rehabilitation Visualization Tool}

Our initial discussions with falls experts revealed that fallers vary widely in their ability to do the exercises prescribed. Therefore, they advised that the users should be able to do the exercises based on their individual abilities, as they would still be able to improve their muscle strength and balance. For this reason, the falls VT did not include visual targets for range of motion. Instead, the users were encouraged to compare their movements as shown by the user mannequin to the guide mannequin in order to ensure that they did the movements correctly (Fig 2).

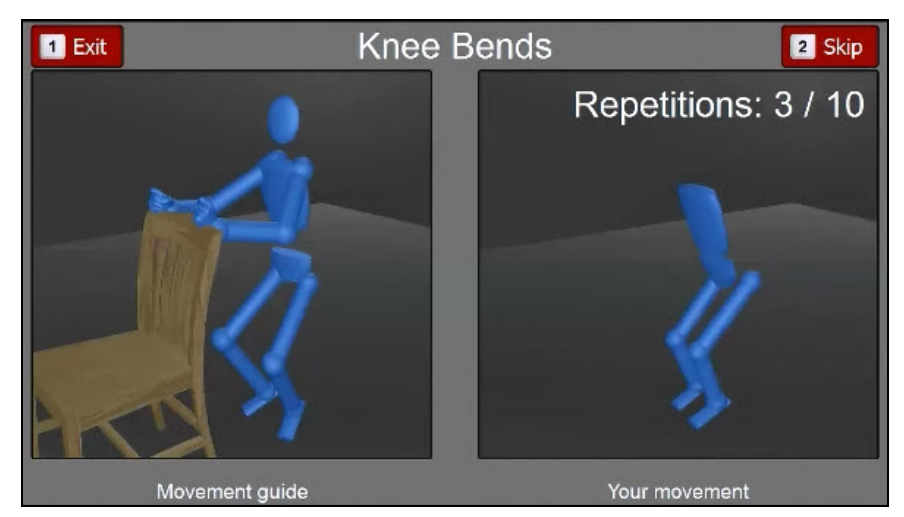

Fig. 2. Falls Visualization Tool: Knee Bend Exercise. Guide mannequin on the left and real time visual feedback on the right

We required a minimum of 6 sensors to animate the human model accurately for falls, as each major body segment tracked required a sensor. Whenever possible we try to minimize the number of sensors a user must wear, however six was the minimum number of sensors needed in order to capture and then visualize the user's movement in a usable way for the end user for falls. These sensors were strapped to the legs, chest, and pelvis in order to provide the feedback necessary to show the correct movements for all of the exercises. Navigation around the software was done through the use of keyboard presses only (key ' 1 ' was used to exit to the main menu; key ' 2 ' to go back to a previous exercise or skip instructions; and key ' 3 ' to go to the next exercise). Keyboard sticker labels were placed on these keys in order to make it easier to find these keys.

\subsection{Knee Replacement Visualization Tool}

The aims of knee replacement rehabilitation are to recover as full a range of motion as possible for the replaced joint and recover muscle strength. Following discussions with orthopaedic surgeons and physiotherapists, we therefore included targets for the users to achieve in the knee replacement VT. However, an overlaid ghost movement was not used because it could give rise to a situation whereby a user in the early stages of recovery could over extend or flex while trying to keep up with the range of movement and pace which can lead to injury. Another unique feature of the knee 
rehabilitation VT is that it gives real-time feedback to only the operated leg although the whole body is shown - to provide guidance on correct posture. This focus on the operated leg allows us to reduce the number of sensors needed to two - one above and one below the knee. This allows for an easier setup in the home. A graphic with a gradient of colors (red to green) was included to show the users the range of motion achieved during each repetition: a deep green represented the recommended range of movement; a light green represented an 'almost there' range; a yellow indicated a minimum range and a red indicated a poor range. For instance, Fig. 3 shows a typical visual feedback.

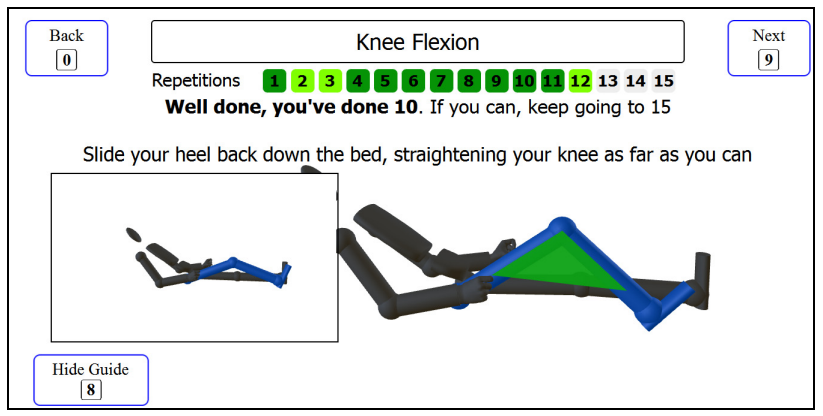

Fig. 3. Knee Replacement Visualization Tool: Knee Flexion Exercise. Guide mannequin in inset and real time visual feedback in the background

The colored repetition icons were also included to show how well each repetition was done. To keep a user motivated, messages such as "Well done, you've done 10, try to keep going to 15 " is shown under the repetition counter. Unlike the falls VT, we opted for a simpler way to show the guide mannequin by making it show only when the user needs it. This way we reduced the cluster on the screen. The users interacted with the knee replacement VT using a remote control similar to a TV remote to facilitate ease of use. The visualization buttons and options were mapped to single numeric keys on the remote control to ensure that the VT can easily be manipulated from a comfortable position.

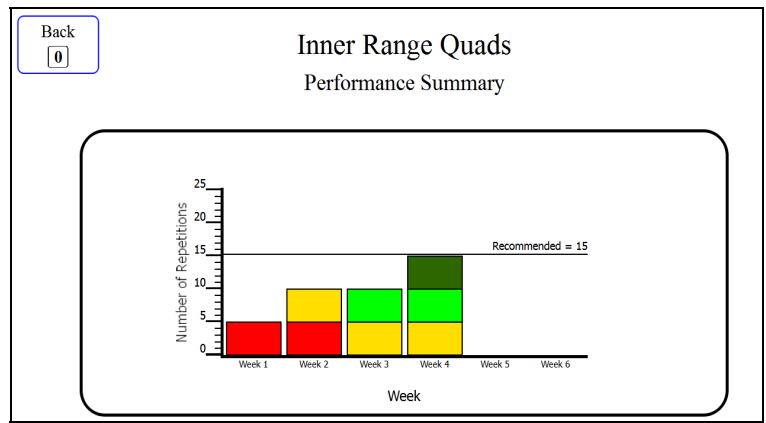

Fig. 4. Knee Visualization Tool: Weekly Progress Charts for Inner Range Quad Exercise 
Progress charts were included in the knee replacement VT in order to track the users' quality of motion in the affected limb over an extended period of time (Fig 4). The color-coded bar chart shown in Fig.4. shows the quality of the repetitions recorded. This way, the user and the Allied Health Profession (AHP) could tell how much progress they had made with each exercise in terms of the ability to move the affected joint and the average number of repetitions completed per week. Both the target user and the AHP participants in our design workshops suggested that progress should be shown on a weekly basis rather than a daily basis because not every exercise session will show progress. AHPs will have remote access to VT midway into the rehabilitation period (clinical follow up) and if there is consistent deterioration in performance or number of repetitions, then urgent medical attention will be provided.

\section{$4 \quad$ Methodology}

Four usability studies were carried out with fallers and former knee replacement patients in the laboratory and in the home - in order to answer the following research questions:

- How easy is it for the users to use the motion sensors (strapping on and operating)?

- Does the use of visualizations enable the user to perform the rehabilitation exercises more correctly in comparison to the standard care exercise booklets?

- Are these visualization tools acceptable and usable by seniors?

The laboratory studies were undertaken first because we could control the environment e.g. setup, layout, interruptions and other health and safety variables. Once we had undertaken the laboratory studies and had eliminated any design issues that caused the users concerns we proceeded to the home studies.

\subsection{Participants}

University ethics was obtained for the studies. We recruited seniors aged 60 years and above for all four of the usability studies. Some of the participants were recruited by telephone, or had attended the previous design workshops we had undertaken one year before. Written consent was obtained from each of our participants. For the knee replacement studies, all of the users had their knee replaced in the past 18 months. For the falls studies, the participants had all had at least one fall in the 12 months prior to the usability studies.

Table 2. Distribution of Participants

\begin{tabular}{lll}
\hline Group & Laboratory & Home \\
\hline Fallers & 3 participant (1 female, 2 males, & 2 participant (1 female, 1 male, \\
& mean age 68) & mean age 79) \\
Knee Replacements & $\begin{array}{l}\text { 3 participants (3 males, mean } \\
\text { age 71) }\end{array}$ & $\begin{array}{l}\text { 3 participants (1 female, 2 males, } \\
\text { mean age 63) }\end{array}$ \\
\hline
\end{tabular}


Their educational backgrounds were varied and so was their experience with computers - e.g. out of the 11 participants, 3 had never used a computer. Table 2 shows the distribution of participants.

\subsection{Procedure}

In the laboratory studies, the furniture was arranged in the laboratory to simulate a home environment with all the items necessary to carry out home exercises - a table to place a television and a laptop computer on; and a chair to do the exercises with (Fig 5). The participants were encouraged to think aloud while doing the tasks given.

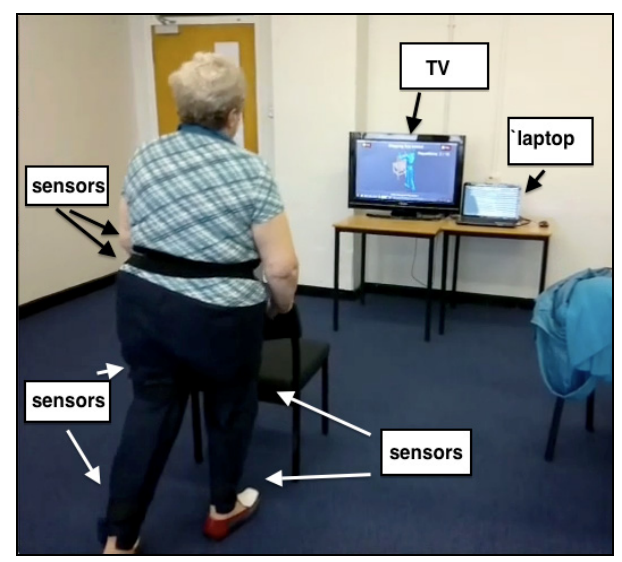

Fig. 5. Laboratory Setup: A participant using the Fallers VT for an exercise requiring the use of a support

The second sets of studies were then carried out in users' homes in order to evaluate these visualization tools in their intended environment (Fig 6).

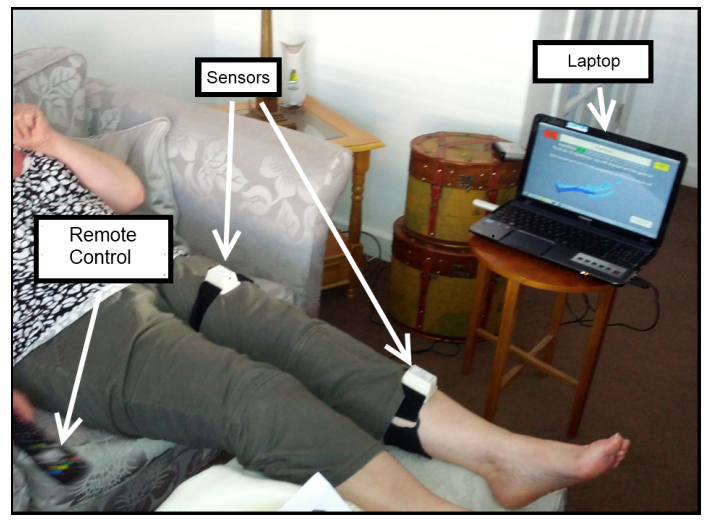

Fig. 6. Home Setup: A participant using the Knee VT 
In both the falls and knee replacement studies, we used a within-subjects design because we believed that using the same participant to test the usability of both the exercise booklet and visualization would produce a more accurate comparison as each of the participants would have experienced the two types of rehabilitation aid first hand. Another merit of the within subject test design is that we can maximize the potential of our outcomes given the small number of participants.

The main procedure for the studies was divided into phases, each containing tasks, which were intended to investigate various aspects of the use of both standard care as well as the visualization tools. These phases are shown in tables 3 and 4 below:

Table 3. Main phases in the Falls studies

\begin{tabular}{ll}
\hline Phases & Procedure \\
\hline Phase 1 & $\begin{array}{l}\text { Participants were asked to perform } 3 \text { exercises using the booklets (the exercis- } \\
\text { es were the same for all of the participants) }\end{array}$ \\
Phase 2 & $\begin{array}{l}\text { Participants were asked to put on the sensors using instructions from the visua- } \\
\text { lization tool only } \\
\text { Participants were asked to perform } 3 \text { exercises using the visualization tool (the } \\
\text { same exercises done in phase 1) }\end{array}$ \\
\hline
\end{tabular}

Table 4. Main phases in the Knee Replacement studies

\begin{tabular}{ll}
\hline Phases & Procedure \\
\hline Phase 1 & $\begin{array}{l}\text { Participants were asked to perform } 3 \text { exercises using either the booklet or } \\
\text { visualization (the exercises were different for all of the participants) }\end{array}$ \\
Phase 2a & $\begin{array}{l}\text { A short training session was provided to the participants on how to use the } \\
\text { visualization tool }\end{array}$ \\
Phase 2b & $\begin{array}{l}\text { Participants were asked to put on the sensors using instructions from the train- } \\
\text { ing, user manual or the visualization tool }\end{array}$ \\
Phase 3 & $\begin{array}{l}\text { The participants were asked to perform } 3 \text { exercises using the visualization or } \\
\text { the booklet (same exercises done in Phase } 1 \text { but with a different tool). }\end{array}$ \\
\hline
\end{tabular}

Given the factors above, a qualitative and quantitative approach was used to develop the procedure involving the different tasks and short breaks were given at the end of each phase - each phase lasted between 10 and 20 minutes. At the end of each phase, participants were asked to complete short questionnaires, and semi-structured interviews on the tasks undertaken.

In phase 1, the participants were asked to do a few exercises with the booklet, while being observed. Two exercise booklets commonly used for home rehabilitation were used: a) The 'Age UK' exercise booklet for the falls studies [28] and b) The Joint Pathways knee rehabilitation exercise booklet for the knee replacement studies [27]. In phase 2, the participants were asked to follow instructions on the screen while they strapped the sensors onto the various limb segments. We wanted to investigate how easy the task was for them, as well as how well the instructions described the management of these sensors. We also noted how long it took them to put on the 
sensors. In phase 3, the participants were asked to do some exercises with the visualization tool, and provide feedback on their experiences with these tools.

The sensor calibration procedure used in the falls studies required the users to stand upright for 5 seconds before each exercise. An extra 'training' phase was included in the knee replacement studies because there was a more complex sensor calibration procedure used - than the one used in the falls studies -this was because for knee replacement we need a reference angle to provide accurate feedback on the knee's range of movement. The calibration procedure included putting a foam wedge with a known angle under the knee.

In the falls studies, the participants did the exercises with the booklet first before using the visualization tool. There were two main reasons for this - first, the participants had all used a booklet in the past for falls rehabilitation. Secondly, it was expected that because the visualization tool contained animated movements that demonstrated recommended pace - unlike the booklet - using it before the booklet could affect the way the participants carried out an exercise using the booklet. As regards the participants in the knee replacement studies not all of them had used the knee replacement booklet in the past. Therefore, to minimize the potential transfer of learning by using either the exercise booklet or visualization first, the order of the use of the two tools was randomized among the participants with 3 of the participants using the booklet first while the other 3 used the visualization tool first.

\section{$5 \quad$ Key Observations and Findings}

In both the falls and knee studies, a facilitator closely observed the participant as the participant carried out the tasks in the various phases. In addition we took notes and timed the users during the tasks. In this section, we report on our quantitative and qualitative findings for both the home and laboratory tests because the results were broadly similar.

\subsection{Performance of Rehabilitation Tools}

\section{Falls Rehabilitation}

We observed that with the standard exercise booklet the participants performed the repetitions in each exercise at a quick, but roughly uniform pace. With the visualization tool (VT), the repetitions were initially quicker (during the first few repetitions), and then slower and more regular afterwards. Figure 7 shows the difference in average time per repetition for the 'knee bends' exercise. The results were similar for the other exercises, with the participants performing the repetitions more slowly and in a more controlled manner while using the visualization tool.

When asked to comment on using the visualization tool for their exercises, the participants' opinions were similar across both the laboratory and home studies. The main responses were that: 


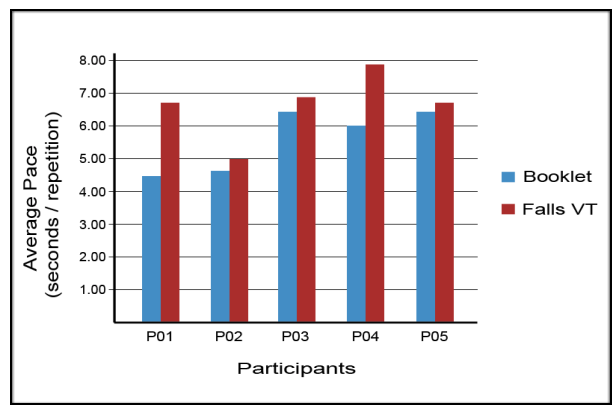

Fig. 7. Average Repetition-pace for the Knee Bends exercise

1. They tried to follow the movements as shown by the guide mannequin; the result of this was that they started to slow their movements down after a few repetitions.

2. They concentrated on the guide mannequin and not on the user mannequin i.e. the mannequin that showed their own movement, for most of the time. However when we further explored this observation with them, they said they would start to concentrate more on their own movement feedback as their confidence and understanding of the system grows.

3. They liked the ability to see the user mannequin that showed their own movement.

4. They all felt that they would use both the booklet and visualization tool if given the chance, but would use the visualization tool more, as they found the feedback helpful and important to improving their confidence in the exercise program.

\section{Knee Replacement (KR) Rehabilitation}

The findings were similar for the knee studies in terms of quality of movement, with the visualization tool encouraging slower, more controlled movements. Fig. 8 shows the Straight Leg Raise exercise and the results shown here shows a typical result for all of the other exercises tested.

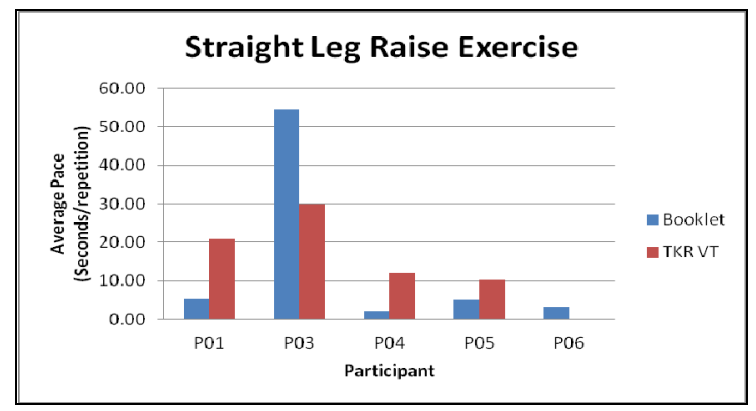

Fig. 8. Average pace for the Straight leg raise exercise

One issue that we observed was with the booklet instruction for the Straight Leg Raise, the instruction asks the patient to raise your leg 6 inches. All of the participants had different concepts of how high 6 inches was. We noted that participants P01, P05 
and P06 had not used the exercise booklet before and participant P06 couldn't complete the exercise with the visualization tool because there was a software bug in the calibration algorithm. As the participants used the booklet, the exercises were performed faster than the recommended pace except for P03 who did the exercise very slowly because he was very talkative and was distracted by waiting for responses from the facilitator. Furthermore, none of the participants managed to keep the hold time for the exercises and lost count of the number of repetitions completed.

On the other hand, we observed that while using the visualization tool, the participants concentrated on their own movement and tried to achieve the green targets. One of the participants in the knee replacement studies forgot his reading glasses at home however, he was still able to use the tool as large font sizes had been used. Another participant brought the laptop computer closer so it could be seen. However, she commented that the elements on the screen could be seen clearly.

When asked to comment on using the visualization tool for their exercises, the participants' opinions were similar across both the laboratory and home studies. The main responses were that:

1. They liked the color-coded fan concept and thought that it helped them to achieve the correct performance of the exercise. One participant commented: "It is very useful to have fullness of each exercise calibrated by color code".

2. Of particular note is P01 who got the instruction for the straight leg raise exercise wrong and was doing something different. He couldn't get into the green zone. He commented as follows "I'm getting this wrong isn't it? I will show the guide. Oh! I was wrong". He was able to correct this without any cue from the facilitator when he viewed the guide mannequin.

3. When asked, "To what degree did the visual feedback help you to perform the rehabilitation exercises?" 5 out of the 6 participants found it very helpful and one participant found it moderately helpful. A typical comment received was "This is a big help because you don't count seconds when doing the static quad on your own. Sometimes you wait too long or too short".

4. All of the participants successfully accessed the progress charts. See Fig. 4. When the participants were asked, "To what degree do you think the progress charts will help you during your home rehabilitation?" Using a Likert scale of $1-5$ with 1 being 'Not helpful' and 5 being 'very helpful'. 2 out of 3 participants said 'very helpful' and 1 participant said 'helpful'. One participant commented, "It is very important to stimulate motivation to continue at an appropriate level". All of the participants found the charts very easy to interpret.

\subsection{Evaluation of the Sensors}

We took into account that the number of sensors required for the falls and knee replacement visualization tools are 6 and 2 respectively. We asked the participants to put on the sensors using the instructions provided on the screen. They were also asked to repeat this task after five minutes (in order to see how well they could remember to put on the sensors on the second attempt). Most of the participants were able to put 
the sensors on correctly on the first and second attempt. Some of the participants in both falls and knee replacement studies put the first sensor on the wrong way (upside down). However, they corrected this when they proceeded to the exercise session and saw a reminder to make sure that the switch was facing upwards, and that they could see the red LED light.

When asked to rate how easy or difficult this task was, 5 of the participants said it was very easy and 4 were confident that they could set up the visualization tools on their own in the home. It should be noted (see Figure 2) that the sensors had been labeled and had to be worn on the front of specific limb segments with the switch facing up. When asked about "how comfortable it is to wear the sensors", all the participants said the sensors were very comfortable to wear for long periods. All the participants also said they were able to follow the on screen instructions to successfully complete their exercises.

The sensors performed well in both the home and laboratory environment but for one participant in the Knee VT group. The reason was that the calibration algorithm relied on facing the north direction and the participant's living room was facing the East, we have since eliminated the requirement for a participant to face in a particular direction.

\subsection{Interaction with the Visualization Tools (Keyboard and Remote Control)}

We investigated 2 setups; the falls visualization used the computer keyboard to navigate through the different visualization options while the knee replacement visualization tool provided two options the keyboard or a remote control. We introduced the remote control as a controller of the tool because of the limited movement of the users in the early stages after knee replacement surgery.

All the participants found navigating the visualization tools using the keyboard easy as the navigation keys were clearly labeled and visible. In the knee replacement studies, the participants preferred the use of the remote control to the keyboard because they didn't have to stand up from the exercise position to operate the tool. All of the participants were able to navigate through the menu items with ease.

\section{Discussion}

\subsection{Home Exercise (Booklet vs. Visualization Tool)}

One of the objectives of the user studies described in this paper was to investigate whether two novel visualization tools could improve the quality of home rehabilitation for falls and knee replacement - compared with the use of standard care. Based on our findings, we identified some issues with the use of booklets (standard care), which makes them less than an ideal solution for effective therapy during rehabilitation.

First, the participants often completed the exercise repetitions too quickly with the booklet. This means that they do not get the therapeutic benefit of a controlled movement. By using the visualization tools, they were able to avoid this problem, as the 
participants followed the movements as demonstrated by the animated mannequins; and were able to achieve the recommended quality of movement.

Secondly, the participants missed some repetitions with the booklet because they had to rely on their memory to keep track of the repetitions. Both the quality and the quantity of physiotherapy exercises received influence the recovery of function, range of movement and muscle strength. This did not happen when they used the booklet but did happen when they used the visualizations, as they were guided through each repetition and there was a repetition counter.

Thirdly, the visualizations showed the participants their movements during each exercise (something that the booklet cannot do) and this gave them more confidence in the exercise programme.

Fourthly, showing two contrasting animations side by side resulted in the users focusing on one and missing out some frames in the other. This in the rehabilitation context is not a bad thing as long as the most important window is emphasized and held in focus. For the Falls VT, it was the correct movement pattern that was important which the users rightly tried to imitate while for the Knee VT the more important image was bigger than the guide mannequin and had changing color graphics that users held in focus. The ability to turn off the guide mannequin was also a useful future that helped users concentrate on a particular window.

Fifthly, the ability to see progress over time was considered a major motivating factor in continuing with the home exercise programme. In the past, the people had used subjective means to measure their progress - e.g. amount of pain in the joint, the ability to stand up quicker, completion of exercise diaries, etc. However, these are subjective and cannot easily be tracked over time. Having objective information about progress over time could provide insights into the effectiveness of standard physiotherapy exercises. In addition, anxiety regarding recovery could be reduced if a user could objectively see that they are capable of greater movement.

\subsection{Usability of Visualization Tool}

Our findings suggest that seniors are capable and able to use technology for their home rehabilitation. In this section we highlight some of the design issues found during our studies and how these could contribute to our understanding of the issues surrounding acceptance of technology for rehabilitation in the home.

Convenience and ease of setup: most seniors are used to operating technologies like the television. Using this metaphor in the development of home rehabilitation technology enabled seniors to feel more confident in their abilities to use a new technology. An acceptable technology should take only a couple of minutes to start and the menu easily accessible via limited numeric and arrow keys. The majority of the participants were able to set up the exercise sessions and correctly calibrate the sensors using the instructions in the visualization tools.

Initial Training and clear onscreen Instructions: An initial training session in the home environment that includes a practical walkthrough of how to use a new visualization technology increases the likelihood of its acceptance. Furthermore, because some seniors are not familiar with some technical jargon such as USB these terms 
should be avoided. If these terms are to be used in such a tool, it should be explained in a straightforward way so that the users understand it better. Memory is known to decline with increasing age. Therefore, it is important to provide instructions that are able to give the users the right information as soon as possible.

Accessibility: It was important for us to ensure that the visualizations were clear and visible, taking into account eyesight problems - which are common in the elderly population. As highlighted in the findings, the use of larger font sizes, blank spacing, red, yellow and green colors in close proximity was well received - seniors appreciate bright and vibrant colors most especially when it provides information about correct performance of an objective.

\section{Conclusion and Future Work}

The main function of musculoskeletal rehabilitation exercises is to recover impaired motor function in muscles and joints in the lower body. In the home setting, the lack of adherence to rehabilitation could have serious implications for the health and independence of seniors. Furthermore, if the recommended quality of motion (range and pace of movement) is not observed for these exercises, the seniors will probably not obtain the full recovery benefits offered by the rehabilitation programme(s). In this work, we evaluated two novel visualization tools with fallers and knee replacement patients; and compared the use of these tools to standard rehabilitation care (booklets) in the laboratory and in the home. One of the main objectives of this research was to investigate how useful the visualization tools were (versus standard care) in encouraging the recommended quality of motion for the exercises - thereby promoting the maximum therapeutic value of these exercises.

Based on our findings we argue that by using booklets to exercise, seniors may not observe the recommended quality of movement. The main findings supporting this are a) the inability to observe the correct pace of movement from static diagrams and b) the seniors could not tell whether they were doing the exercises the correct way. By using the visualization tools, the seniors were able to overcome these limitations, thereby getting the most out of the exercises from a therapeutic standpoint. The seniors felt that the ability to observe the correct movements - as well as receive feedback on their movements and progress - during rehabilitation was essential to increasing their confidence and motivation to exercise. Furthermore, based on the feedback from our participants, these visualization tools were easy to use and acceptable for home rehabilitation use. Another objective of this study was to investigate the usability of the body-worn sensors necessary to interact with the visualization tools. Our findings suggest that the seniors found the sensors easy to use and that they were confident in their ability to use and manage (e.g. placement on the body, charging, etc.) them without the assistance of a professional.

The study results are limited by the number of participants and the amount of time that the participants had to interact with the visualization tools. The effectiveness of these visualization tools in improving adherence to home rehabilitation programmes over an extended period of time still needs to be assessed. This is the basis for our future work, as we are now deploying these tools in home based pilot randomized controlled studies for $6-12$ weeks. 
Acknowledgements. Funding for this study comes from the Medical Research Council, UK, LLHW (Phase 2), Grant ID: 91021.

\section{References}

1. Woolf, A.D., Pfleger, B.: Burden of major musculoskeletal conditions. Bull. World Health Organ. 81, 646-656 (2003)

2. Scottish Arthroplasty Project Biennial Report 2012, NHS National Services Scotland, p. 9, http://www.arthro.scot.nhs.uk/Reports/sap_national_report_20 $12 \cdot \mathrm{pdf}$

3. Skelton, D., Todd, C.: What are the main risk factors for falls among older people and what are the most effective interventions to prevent these falls? Copenhagen, WHO Regional Office for Europe (2004),

http: / / www . euro. who. int/document/E82552.pdf

4. Thomas, S., Mackintosh, S., Halbert, J.: Does the 'Otago exercise programme' reduce mortality and falls in older adults?: a systematic review and meta-analysis. Age Ageing 39(6), 681-687 (2010)

5. Applegate, W.B., Miller, S.T., Graney, M.J., Elam, J.T., Burns, R., Akins, D.E.: A Randomized, Controlled Trial of a Geriatric Assessment Unit in a Community Rehabilitation Hospital. New England Journal of Medicine 322, 1572-1578 (1990)

6. Medical Advisory Secretariat: Physiotherapy rehabilitation after total knee or hip replacement: an evidence-based analysis. Ontario Health Technology Assessment Series 5(8) (2005)

7. Nyman, S.R., Victor, C.R.: Older people's participation in and engagement with falls prevention interventions in community settings: An augment to the Cochrane systematic review. Age Ageing (2011), http://www.ncbi.nlm.nih.gov/pubmed/21875865

8. Pisters, M.F., Veenhof, C., Schellevis, F.G., Twisk, J.W.R., Dekker, J., De Bakker, D.H.: Exercise adherence improving long-term patient outcome in patients with osteoarthritis of the hip and/or knee. Arthritis Care \& Research 62, 1087-1094 (2010)

9. Scott, J.C.: Osteoporosis and hip fractures. Rheumatic Diseases Clinics of North America 16(3), 717-740 (1990)

10. Fessel, K., Nevitt, M.: Correlates of fear of falling and activity limitation among persons with rheumatoid arthritis. Arthritis Care and Research 10(4), 222-228 (1997)

11. Button, K., Iqbal, A.S., Letchford, R.H., Van Deursen, R.W.M.: Clinical effectiveness of knee rehabilitation techniques and implications for a self-care treatment model. Physiotherapy 98, 287-299 (2012)

12. Wheeler, J.W., Shull, P.B., Besier, T.F.: Real-Time Knee Adduction Moment Feedback for Gait Retraining Through Visual and Tactile Displays. Journal of Biomechanical Engineering 133, 041007 (2011)

13. Walker, C., Brouwer, B.J., Culham, E.G.: Use of visual feedback in retraining balance following acute stroke. Phys. Ther. 80, 886-895 (2000)

14. Zhou, Hu, H.: Human motion tracking for rehabilitation-A survey. Biomedical Signal Processing and Control 3, 1-18 (2008)

15. Uzor, S., Baillie, L., Skelton, D., Fairlie, F.: Identifying barriers to effective user interaction with rehabilitation tools in the home. In: Campos, P., Graham, N., Jorge, J., Nunes, N., Palanque, P., Winckler, M. (eds.) INTERACT 2011, Part II. LNCS, vol. 6947, pp. 3643. Springer, Heidelberg (2011) 
16. Suttanon, P., Hill, K.D., Said, C.M., Byrne, K.N., Dodd, K.J.: Factors influencing commencement and adherence to a home-based balance exercise program for reducing risk of falls: perceptions of people with Alzheimer's disease and their caregivers. International Psychogeriatrics 24, 1172-1182 (2012)

17. Lim, C.K., Chen, I.-M., Luo, Z., Yeo, S.H.: A low cost wearable wireless sensing system for upper limb home rehabilitation. In: 2010 IEEE Conference on Robotics Automation and Mechatronics (RAM), pp. 1-8 (2010)

18. Doyle, J., Bailey, C., Dromey, B., Scanaill, C.N.: BASE - An interactive technology solution to deliver balance and strength exercises to older adults. In: 4th International Conference on Pervasive Computing Technologies for Healthcare, PervasiveHealth, pp. 1-5 (2010)

19. Harms, M.: Advancing technology in rehabilitation. Physiotherapy 98, 181-182 (2012)

20. Tanaka, K., Parker, J., Baradoy, G., Sheehan, D., Holash, J.R., Katz, L.: A Comparison of Exergaming Interfaces for Use in Rehabilitation Programs and Research. LDG 6, 69-81 (2012)

21. Gerling, K.M., Schulte, F.P., Masuch, M.: Designing and evaluating digital games for frail elderly persons. In: Proc. of ACE 2011 (2011)

22. Borghese, N.A., Pirovano, M., Mainetti, R., Lanzi, P.L.: An integrated low-cost system for at-home rehabilitation. In: 2012 18th International Conference on Virtual Systems and Multimedia, VSMM, pp. 553-556 (2012)

23. Theng, Y.-L., Dahlan, A.B., Akmal, M.L., Myint, T.Z.: An exploratory study on senior citizens' perceptions of the Nintendo Wii: the case of Singapore, pp. 1-5. ACM, Singapore (2009)

24. Fung, V., Ho, A., Shaffer, J., Chung, E., Gomez, M.: Use of Nintendo Wii Fit ${ }^{\mathrm{TM}}$ in the rehabilitation of outpatients following total knee replacement: a preliminary randomised controlled trial. Physiotherapy 98, 183-188 (2012)

25. Ziefle, M., Röcker, C.: User Diversity as a Challenge for the Integration of Medical Technology into Future Smart Home Environments. In: Human-Centered Design of E-Health Technologies, pp. 95-126. IGI Global, USA (2010)

26. SilverFit, http://www.silverfit.nl/en.html

27. JointPathways ${ }^{\mathrm{TM}}$ Patient Guides for Knees, Golden Jubilee National Hospital NHS Scotland

28. Age UK. Preventing Falls: Strength and balance exercises for healthy ageing (2011), http : / / www . ageuk . org. uk/Documents / EN-GB / ID8950\%20

Strength\%20And\%20Balance 20 Book.pdf?dtrk=true

29. Hondori, M.H., Khademi, M., Lopes, C.V.: Monitoring Intake Gestures using Sensor Fusion (Microsoft Kinect and Inertial Sensors) for Smart Home Tele-Rehab Setting (2012),

http://www.isr.uci.edu/ mkhademi/

files/Publications/HIC2012.pdf

30. Bo, A.P.L., Hayashibe, M., Poignet, P.: Joint angle estimation in rehabilitation with inertial sensors and its integration with Kinect. In: 2011 Annual International Conference of the IEEE Engineering in Medicine and Biology Society, EMBC, pp. 3479-3483 (2011)

31. Uzor, S., Baillie, L., Skelton, D.: Senior designers: empowering seniors to design enjoyable falls rehabilitation tools. In: Proceedings of the 2012 ACM Annual Conference on Human Factors in Computing Systems, pp. 1179-1188 (2012)

32. Loudon, D., Macdonald, A.S., Carse, B., Thikey, H., Jones, L., Rowe, P.J., Uzor, S., Ayoade, M., Baillie, L.: Developing visualisation software for rehabilitation: Investigating the requirements of patients, therapists and the rehabilitation process. Health Informatics Journal 18, 171-180 (2012) 